http://jmscr.igmpublication.org/home/

ISSN (e)-2347-176x ISSN (p) 2455-0450

crossref DOI: https://dx.doi.org/10.18535/jmscr/v8i3.03

Journal Of Medical Science And Clinical Research

\title{
Pectoralis Major Myo-cutaneous Flap Work-Horse In Reconstruction of Oral Cavity Cancers
}

Authors

\section{T.Mohan Vijay Kumar, Guduru Sumanth, Kaushik Hari Mch (Surgical Oncology)}

\begin{abstract}
Today, in the era of microvascular reconstructive surgery is well-grounded in the vernacular of microvascular surgeon as well as the increasingly more educated public. One of the concepts in head and neck reconstruction surgery is that whenever possible, one should try to reconstruct the defects with tissues that more closely resemble the missing part not only in color but also in thickness and the texture.

Equally important in the reconstructive is to keep in mind the needs of our subjects and their consent to undergo a more extensive reconstruction surgery using free tissue transfer. In those cases, as well as those where the free grafts transfer has failed, the use of pedicled local or regional flaps is an essential aspect of the armamentarium of reconstructive surgeons.

Though free flaps are presently the gold standard for head and neck reconstruction, PMMC flaps remain a robust, versatile pedicled workhorse flap used for a variety of head and neck reconstructions with a comparatively easier learning curve and a shorter operating time to ease the enormous workload in our hospitals.

In this article, we have reviewed 30 head and neck cancer cases, which we had done in the Surgical oncology department of Nri Medical College, Chinna Kakani in which we have used the PMMC flap as a reconstructive tool for various defects produced after resection of head and neck malignancies. These include buccal mucosal cancers, cancer of the floor of mouth, tongue, retromolar trigone, lip, mandible, and parotid gland.

We did not have even one case of total flap necrosis. However, minor complications like partial flap necrosis, infection, wound dehiscence, and the oro-cutaneous fistula was present in a few of our cases.

We thus conclude that PMMC flap remains as one of the main flaps for salvage reconstruction secondary to the loss of the microvascular flaps as well as in those patients in whom microvascular flaps are either contraindicated or cautioned due to existing comorbidities that diminish their ability to tolerate an extended operating time.
\end{abstract}

\section{Introduction}

The initial description of the pectoralis major flap of head and neck reconstruction was Ayrian in 19th century. The following years he published his work in the Journal of the Plastic and Reconstructive Surgery. ${ }^{1}$

Since the description of this flap, this use quickly became widespread, and within a short span, it was flap of choice in head and neck cancers for reconstruction. The pectoralis muscle flap held its position as workhorse flap of defects in the head and neck cancers for many years until the introduction of the radial forearm free flap in recent years.

The location of donor site as it relates to the head and neck makes this flap an excellent option for 
defects in this region. The harvest of pmmc flap can be carried out with the patient in a supine position, i.e., in the same place as the ablative head and neck operation. The option for a twoteam combined approach is also possible, although the surgical field would be slightly over crowded.

One of the advantages of the pectoralis major myocutaneous flap is the quality and quantity of tissue that can be harvested. The pectoralis significant muscle enables the closure of a multitude of defects in the head and neck, but its robust amount allows for the coverage of the reconstruction plates used in the mandibular surgery, therefore decreasing the chance of plate exposure through the skin or the mucosa. At the same time, the muscle coverage in the neck provides additional pro- tection of the great vessels. This fact is of importance in patients who have had a radical neck or a type I or II mod-ified radical neck dissection. The coverage of the vessels becomes even more significant in those patients needing adjuvant radiation therapy or those who have already received radiotherapy and may be faced with delayed wound healing.

\section{Materials and Methods}

Thirty patients with head and neck malignancies were operated from 2015 to early 2018, where PMMC flap reconstruction was performed. All the operations were done at Medical College, Kolkata. A clearance from the ethical committee was obtained for this study. Every patients gave written \& informed consent for surgery and also consent for preoperative, operative, and postoperative photographs to be produced for this study and further publication.

For this study, the demographic details of the patients were recorded along with the pathological stage of disease in which they presented to us. Also, the comorbidities of the patients, any previous treatment is taken, the surgical resection procedures are undertaken, and the post-operative complications either flap related or not, we're all recorded. Ipsilateral PMMC flap was harvested in each case using the standard techniques and was used for reconstruction after resection of the lesion.

\section{Results \& Analysis}

Out of 30 patients, 24(80\%) were male, and $6(20 \%)$ were female. Eight patients $(26.67 \%)$ out of 30 were in the $7^{\text {th }}$ decade of life, $18(60 \%)$ in the $5^{\text {th }}$ and $6^{\text {th }}$ decades, and two each $(6.67 \%)$ in the $3^{\text {rd }}$ and $4^{\text {th }}$ decades. Twenty-seven patients $(90 \%)$ had malignancy in the oral cavity, 2 with malignancy in the base of tongue i.e., oropharynx $(6.67 \%)$ and 1 with adenoid cystic carcinoma of the parotid gland (3.33\%). All the malignancies in the oral cavity and oropharynx were squamous cell carcinoma. 3 out of those 30 cases $(10 \%)$ were recurrence where PMMC flap was used after resection of the recurrence to cover the defect. Two of the three recurrent lesions were from outside Medical College, Kolkata, who had primary closure after excision of the primary lesion. One patient was our own follow up developed recurrence \& did not receive radiotherapy after excision of the primary tumor and modified radical neck dissection with histopathologically confirmed negative margins and metastatic cervical lymph nodes. Out of the 27 oral cavity cancers, $12(40 \%$ of total) were in the buccal mucosa and three each $(10 \%)$ in the floor of the mouth, tongue (within anterior twothird), retromolar trigone. Two patients $(6.67 \%)$ had a lesion in the lip and four patients (13.33\%) in the mandible.

Only 6 cases $(20 \%)$ reported with early disease i.e., stage I and II of the disease. Twelve patients (40\%) reported with stages III disease and 12 with stage IV. All patients were undertaken for operation without prior radiotherapy. PMMC flap reconstruction was performed in all cases as the primary procedure. Temporary tracheostomy had to be done in 10 cases $(33.33 \%)$, which were weaned off during follow up in all the 10 cases that required it.

Post-operative complications were categorized under two broad headings i.e., flap related and 
flap unrelated. No perioperative deaths occurred. Eighteen patients $(60 \%)$ had uneventful recovery without any complication. Flap unrelated complications like a chest infection, superficial thrombophlebitis developed in a few patients who were not major, and did not prolong hospital stay. Flap related complications: None of the patients had total flap necrosis. Six patients $(20 \%)$ had partial flap necrosis, of which 2(6.6\%) were major i.e., full-thickness. Four patients (3.33\%) had marginal or superficial flap necrosis. Other flap related complications were wound dehiscence, oro-cutaneous fistula, infection, etc. Donor site wound dehiscence developed in 3 patients (10\%). None of these patients with complications required any salvage procedure. They all gradually recovered completely with regular dressing and conservative approaches though this prolonged the hospital stay and increased the cost of treatment. Overall cosmetic outcome was satisfactory in these patients. All the patients were referred for radiation therapy and asked to follow up in our OPD at regular intervals.

One patient (3.33\%) who had a buccal mucosal growth close to the mandible and excised with $1 \mathrm{~cm}$ margins \& neck dissection and which was reconstructed at the index operation by scalp and forehead flaps, developed recurrence and was taken up for re-resection. That patient had to undergo a second reconstructive procedure using the PMMC flap from the ipsilateral side. Now he is doing fine.

\begin{tabular}{|c|c|c|}
\hline Age group & No. of patients & Percentage (\%) \\
\hline $31-40$ & 2 & 6.67 \\
\hline $41-50$ & 2 & 6.67 \\
\hline $51-60$ & 9 & 30 \\
\hline $61-70$ & 9 & 30 \\
\hline $71-80$ & 8 & 26.67 \\
\hline
\end{tabular}

\begin{tabular}{|c|c|c|}
\hline Primary site & No. of patients & Percentage(\%) \\
\hline Oral Cavity & & \\
\hline Buccal mucosa & 12 & 10 \\
\hline Floor of mouth & 3 & 10 \\
\hline Anterior two-thirds of Tongue & 3 & 10 \\
\hline Retro-molar trigone & 3 & 6.67 \\
\hline Lip & 2 & 13.33 \\
\hline Mandible & 4 & 6.67 \\
\hline Oro-pharynx & 2 & 3.33 \\
\hline Base of tongue & & \\
\hline Parotid gland & 1 & \\
\hline
\end{tabular}

\begin{tabular}{|c|c|}
\hline Complications & No. of patients \\
\hline Flap related & \\
\hline Total flap necrosis & NIL \\
\hline artial flap necrosis Major i.e., full-thickness & 2 \\
Minor i.e., marginal/superficial & 4 \\
\hline Others & 4 \\
Oro-cutaneous fistula & 7 \\
Wound dehiscence & 6 \\
Infection & 3 \\
\hline Donor site wound dehiscence & eew (insignificant) \\
\hline Flap unrelated & 18 \\
\hline Uneventful recovery & NIL \\
\hline Peri-operative death &
\end{tabular}

\section{Discussion}

Currently, free flap reconstruction is undoubtedly the first choice for head and neck cancers providing one stage restoration with better cosmetic and functional results (Ref.1). However, even in the hands of experienced microsurgeon total flap necrosis occurs in free flap reconstructions; but total flap loss in PMMC flap is uncommon (Ref.2). This fact, coupled with the relatively short learning curve for PMMC, makes it a desirable flap for head and neck reconstruction in a developing country like India with limited medical resources and a huge patient load, which demands a robust flap. It would utilize fewer resources and could be accomplished as a single team approach with relatively fast flap harvest and inset, thereby avoiding the logistics of getting two teams to work together always (Ref.3). PMMC is a flap which can be used not only as a flap for primary constructions of head and neck defects but also as a salvage procedure in case of free flap necrosis and in patients were free to flap reconstructions are contraindicated as in cases with inadequate recipient vessels and medical comorbidities making the patient unfit for a long surgery. It can also be used with a free flap to cover very large soft tissue defects overlying major vessels (Ref.1,2).

The Pectoralis Major is fan-shaped muscle that helps in adduction and internal rotation of the arm. It has four sites of origin- clavicle/ the manubrium and sternum extending inferiorly to where the 6th and 7th costal cartilages are attached/ the costal cartilage from second to sixth ribs and the aponeurosis of the external oblique muscle. From this wide origin, the muscle inserts into the crest 
of the greater tubercle of the humerus. It is a type 5 muscle, according to Mathews Nahai classification. The main blood supply comes from the thoracoacromial artery, which is a branch of the second part of the axillary artery, and it divides into four branches - pectoral, clavicular, acromial, and deltoid. pectoral branchs of the thoracoacromial artery is the main branch of the sternocostal part of the muscle which descends along the posterior surface of the muscle within the sheet of the muscle and enters the deep surface of the muscle at the midpoint, and some of these vessels pierce the surface of the muscle to supply the overlying skin. The terminal branch of the pectoral artery passes around the inferior-lateral border of the pectoralis muscle to reach the skin. Many of the arterial branches pass medially along the fascial septa and anastomose with segmental anterior perforating branches from the internal thoracic artery. These perforators penetrate the medial part of the pectoralis to reach the overlying skin as a direct cutaneous vessel and thus if the third perforating branch of the internal thoracic artery is taken, it ensures blood supply of the distal part of the skin paddle( especially if the skin paddle is taken caudally and medial to the nipple) due to the choke anastomosis of the internal thoracic and thoracoacromial artery. A further anastomosis may exist with a branch of the lateral thoracic artery which runs along the lateral edge of the muscle

The skin paddle of PMMC flap can be of the following types- inferomedial, lateral, parasternal, extended, bi-paddled, and as an osteomusculocutaneous flap. Placing the flap inferomedial was a common method of reconstruction in our series. Exposure of the muscle was usually achieved by an open approach by a skin incision from the proper skin Island along the anterior axillary fold so that direct access was gained to the lateral margin of the pectoralis muscle. An incision was then made along the proposed marking of the skin paddle, and the dermis of the paddle was fixed to the underlying pectoral muscle by interrupted sutures.
First, the superior flap containing skin and subcutaneous tissue was raised up to the clavicle. .The skin paddle was then lifted from the inferior margin to the superior portion, usually including the third perforating branch of the internal thoracic artery. As the muscle along with skin paddle was lifted from "down-up", the thoracoacromial pedicle was located at the undersurface of the pectoralis major muscle, and this pedicle was followed superiorly, and the muscle trimmed both medially and laterally by cutting it with diathermy, always keeping an eye on the thoracoacromial pedicle so that it was not injured. The flap was then tunneled into the neck over the clavicle keeping in mind to keep the width of the tunnel to at least four fingers so that there is no compression over the pedicle. The sternocleidomastoid muscle was usually resected at the time of neck dissection to accommodate the pedicle of the PMMC flap to reach the oral cavity for the reconstruction of various defects created after resection of different head and neck cancers. (figure 1-6)

When a PMMC and a DP flap were concomitantly required for reconstruction, the DP flap territory was elevated first, keeping the second intercostal artery intact, and this gives access to the lateral margin of the PMMC flap. Then a similar method was employed for the elevation of the PMMC flap.

We have used this flap to reconstruct a variety of defects resulting after wide local excision of cancers of the oral cavity, total glossectomy, and radical parotidectomy.

We have also used PMMC as a salvage flap in 3 cases used to reconstruct defects created after wide local excision of recurrent cancers.

Reported complication rate in various series varies from $17 \%$ to $63 \%$ (Ref. 1,4,5,6). In our study, we did not observe even a single case of fullthickness necrosis of PMMC flap. 20\% (6 patients) had partial flap necrosis, of which two patients( $6.66 \%)$ had partial full-thickness flap necrosis, and $4(13.33 \%)$ patients had only marginal and superficial flap necrosis which is 
comparable to the findings in other studies (Ref.1,5,7,8,9).

One major advantage of PMMC flap is its survival, as total flap loss is extremely uncommon. However, this incidence of necrosis in a free flap, done even by an experienced microsurgeon, is high. Many factors have been suggested for partial flap necrosis like the presence of a random portion of the skin to the distal end of the flap, the flap harvest going beyond the 7 th rib, not creating a wide enough tunnel during the transfer of the flap, preservation versus removing clavicular attachment of pectoralis muscle.

Hence in most cases, we tried to encompass skin perforator vessels that arise from the intercostal branches of the internal thoracic artery by positioning the skin Island just medial to the nipple over the $4^{\text {th }}, 5$ th and 6th intercostal spaces as proposed by Rikimani et al. (Ref.10). The cutaneous vessels are supplied by pectoral branches of the thoracoacromial artery through open choke vessels when the main blood flow through the internal thoracic artery is interrupted during PMMC flap elevation (Ref.10). Thus by including this modification, a total axial myocutaneous flap may be created.

However, if an extended PMMC is done by taking skin Island below the 7th rib, it increases the chance of partial flap loss as this portion of an island will have a random blood supply as they are originally supplied by cutaneous branches of the superior epigastric artery.

Another pitfall relates to lateral pectoral nerve division (Ref.11), as this nerve lies parallel/ oblique to the thoracoacromial pedicle, and so as the flap is rotated through 180 degrees, the nerve becomes taut especially when it runs oblique to the pedicle and thereby presses against the pedicle. Hence this nerve should be dissected and divided when this situation happens (Ref.11).

Other complications include hematoma formation, which developed in no patient in our series. Other series have reported hematoma in $7 \%$ of patients (Ref.7). We attribute this to two factors. We think that it is the extra care we took in continuous suturing the raw margins of the raised pectoralis major muscle with 3-0 vicryl (taking care that the vascular pedicle is not included in the suture) and meticulous hemostasis that we achieved in every stage of the operation; which was responsible for the fact that none of our patients develop this complication.

Fistula developed only in 4 of our patients (13.3\%) and had occurred in only those cases which had some degree of marginal flap necrosis or had diabetes. This figure corroborates with Tripathy et al. (Ref.7), who developed fistula in $12 \%$ of their patients. They had opined that the internal part of PMMC stays in a contaminated environment of saliva and food and consequently is not accessible for mechanical cleansing resulting in infection and fistula. They also took extra care while putting the tripoint suture in the anterior region between flap and mucosa of the floor of the mouth and mucoperiosteum of the cut edge of the mandible.

In our series, all fistula healed spontaneously. Other complications like infection occurred in $6(20 \%)$ of our patients, which is comparable to other studies in the literature.

\section{Conclusion}

- To conclude, we feel that PMMC is still the workhorse flap for head and neck reconstruction with good functional outcomes.

- This is because it has an easy learning curve, a constant and robust vascular pedicle, versatile, and can be done easily in places that have a huge workload and limited resources.

\section{References}

1. Vartanian JG, Carvalho AL, Carvalho SM, Mizobe L, Magrin J, Kowalski LP. Pectoralis major and other myofascial/myocutaneous flaps in head and neck cancer reconstruction: Experience with 437 cases at a single 
institution. Head Neck. 2004;26:1018-23. [PubMed]

2. McLean JN, Carlson GW, Locken A. The pectoralis major myocutaneous flap revisited: A reliable technique for head and neck reconstruction. Ann Plast Surg. 2010;64:570-3. [PubMed]

3. Talesnik A, Markowitz B, Calcaterra T, Ahn C, Shaw W. Cost and outcome of osteocutaneous free-tissue transfer versus pedicled soft-tissue reconstruction for composite mandibular defects. Plast Reconstr Surg. 1996;97:1167-78. [PubMed]

4. Croce A, Moretti A, D'Agostino L, Neri G. Continuing validity of pectoralis major muscle flap 25 years after its first application. Acta Otorhinolaryngol Ital. 2003;23:297-304. [PubMed]

5. Liu R, Gullane $\mathrm{P}$, Brown $\mathrm{D}$, Irish $\mathrm{J}$. Pectoralis major myocutaneous pedicled flap in head and neck reconstruction: Retrospective review of indications and results in 244 consecutive cases at the Toronto General Hospital. J Otolaryngol. 2001;30:34-40. [PubMed]

6. Freeman JL, Walker EP, Wilson JS, Shaw HJ. The vascular anatomy of the pectoralis major myocutaneous flap. Br J Plast Surg. 1981;34:3-10. [PubMed]

7. Tripathi M, Parshad S, Karwasra RK, Singh.Pectoralis major myocutaneous flap in head and neck reconstruction: An experience in 100 consecutive cases. Natl. J. Maxillofac Surg.2015;6(1):37-41

8. Milanovic A, Virag M, Uglesic V, Aljinovic-Ratkovic N. The pectoralis major flap in head and neck reconstruction: First 500 patients. J Craniomaxillofac Surg. 2006;34:340-3. [PubMed]
9. El-Marakby HH. The reliability of pectoralis major myocutaneous flap in head and neck reconstruction. J Egypt Natl Canc Inst. 2006;18:41-50. [PubMed]

10. Rikimaru H, Kiyokawa K, Inoue Y, Tai Y. Three-dimensional anatomical vascular distribution in the pectoralis major myocutaneous flap. Plast Reconstr Surg. 2005;115:1342-52. [PubMed]

11. Cunha-Gomes D, Choudhari C, Kavarana NM. Vascular compromise of the pectoralis major musculocutaneous flap in head and neck reconstruction. Ann Plast Surg. 2003;51:450-4. [PubMed]. 